\title{
Thetic and Categorical, Stage and Individual, Weak and Strong
}

\author{
William A. Ladusaw \\ University of California, Santa Cruz
}

\section{Introduction}

In his classic work on the interpretation of the English existential construction, Gary Milsark (1974) proposed a two-way distinction in interpretations of determiner phrases, a two way classification of one-place predicates, and a generalization about their ability to combine.

Milsark proposed that DPs can be given a 'cardinality' interpretation or a true 'quantificational' interpretation. The distinction between the two types of interpretation, or construal, trifurcates the syntactic category DP into those DPs which are unambiguously quantificational, unambiguously cardinal, or ambiguous between the two construals. The distinction between the two construals is illustrated by a subtle ambiguity in (1-2):

1. Some unicorns entered the garden.

2. Many people were at the party.

He says that "both these examples show an ambiguity in the interpretation of their subject NP. Thus (1) can mean either than an indefinite number of unicoms entered or that some of the unicorns entered, but others, presumably, remained outside. Similarly, (2) can mean either that the party had rather a lot of people at it or that it is true of many people, as opposed, one suspects, to others, that they were at the party." [1974:199] The three-way classification of DPs is exemplified in (3):

3. Weak Construal

$$
\text { a dog, sm men }
$$

this gúy (nondemonstrative)

\section{Strong Construal}

$$
\begin{aligned}
& \text { three, etc. } \\
& \text { several, many } \\
& \text { bare plurals }
\end{aligned}
$$

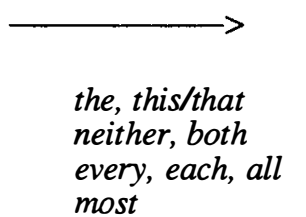

The distinction in one-place predicates divides them into those which express 'state descriptions' and those which express 'properties'. The chart in (4) is part of the extension of this classification:
4. State Descriptions
Properties sick, drunk, open

$$
\text { tall, intelligent, a lawyer }
$$

The constraint on their combination is illustrated in the pattern in (5). 
$\begin{aligned} \text { 5.a } & \text { The man is sick } \\ . b & \text { The man is tall } \\ \text {.c } & \text { Sm men are sick } \\ \text { d } & * \text { Sm men are tall }\end{aligned}$

\author{
(Strong + State Description) \\ (Strong + Property) \\ (Weak + State Description) \\ (Weak + Property)
}

Milsark's Generalization concerns the explanation of (5d):

6. Milsark's Generalization: Properties may only be predicated of Strong NPs. (= Individual level predicates must have strong subjects.)

The terminology which has become standard since Carlson (1977) for the distinction among predicates is, of course, stage-level predicate and individuallevel predicate for 'state-descriptive' and 'property' respectively. The reanalysis of the strong and weak distinction as a division in types of generalized quantifiers was enshrined by Barwise and Cooper (1980).

This much is ancient history. The questions of interest have been:

7.a How can we give a definition of the individual/stage level distinction?

.b What is the distinction between weak and strong construals?

.c Why should Milsark's generalization be true?

To these three questions, I want to add two more:

.d Why are some DPs necessarily weak?

.e Which is the interesting case, $(5 \mathrm{c})$ or $(5 \mathrm{~d})$ ?

That is, is it more interesting that weak readings are possible with stage-level predicates, or that strong readings are necessary with individual-level predicates?

These questions are taken up by Kratzer (1989) and Diesing (1992). Kratzer proposed to define stage-level predicates as those whose meanings contain a bindable spatio-temporal or event variable. From this characterization, a number of traditional tests for the distinction can be interpreted as owing to the success or failure to bind that variable. Diesing builds upon this definition with a series of assumptions from which Milsark's generalization is supposed to follow. As I understand the proposal, its derivation is as in (8):

\section{Diesing's Derivation of Milsark's Generalization}

a. Weak readings of indefinites are the result of existential closure; strong readings involve quantificational operators.

b. VP is the domain of existential closure.

c. The 'subject' role of a predicate is projected VP internally.

d. Individual-level predicates only occur under 'transitive' INFL; stage-level predicates (can) occur under 'unaccusative' INFL.

e. Subjects of raising predicates optionally reconstruct; subjects of control predicates cannot reconstruct.

f. Therefore subjects of Individual-level predicates are subjects of a control predicate (the transitive INFL). 
g. Therefore Subjects of ILPs cannot reconstruct.

h. Therefore Subjects of ILPs cannot be given weak construals.

The last point is presumably equivalent to Milsark's generalization.

\section{Kuroda's Hypothesis}

In a series of papers beginning with (1972) and most recently (1992), Yuki Kuroda has defended the relevance of a distinction in 'judgment forms' which goes back to the philosophy of Franz Brentano $(1874,1924)$ and its application in grammatical theory by his student Anton Marty. To quote Kuroda (1972:154):

"This theory assumes, unlike either traditional or modern logic, that there are two different fundamental types of judgments, the categorical and the thetic. Of these, only the former conforms to the traditional paradigm of subject-predicate, while the latter represents simply the recognition or rejection of material of a judgment. Moreover, the categorical judgment is assumed to consist of two separate acts, one the act of recognition of that which is to be made the subject, and the other, the act of affirming or denying what is expressed by the predicate about the subject. With this analysis in mind, the thetic and the categorical judgments are also called the simple and the double judgments (Einfache Urteil and Doppelurteil).

The most important part of this view for our immediate purpose is that there are two different judgment forms, one of which has a "presupposed" subject in the sense that a precondition for making the judgment is that 'the mind of the judger must be directed first to an individual, before the predicate can be connected to it'. Kuroda's most compelling argument for the relevance of this distinction comes from his analysis of Japanese topicalizing wa as a marker of the logical Subject of a categorical judgment. The analysis can be illustrated by considering the translation of 'the cat is sleeping there' into Japanese as either (9) or (10), which differ only in the postposition on the (determinerless) NP neko.

9. neko ga asoko de nemutte iru

(Thetic) the/a cat there sleeping is

10. neko wa asoko de nemutte iru the cat there sleeping is

(Categorical)

These two sentences reflect the difference in judgment type. (9) expresses a thetic judgment; it would be used to report perception of a situation in which a/the cat is sleeping in a certain place. It simply affirms the existence of an eventuality of a certain type. (10) might be used to describe the same situation, but in an essentially different way. As a categorical judgment it draws attention first to the cat, and then says of the cat that it is sleeping there. Correlated with this difference is the fact that in (10), the bare noun marked by wa cannot be taken as a nonspecific indefinite cat; it must be a particular cat. This follows from the presuppositional nature of the subject of a categorical judgment; the cat must be presented qua individual before the property of sleeping there can be attributed to it.

It is a short step from this discussion to see a parallel between the elimination of the possibility of the nonspecific construal of neko in (10) and the anomaly in 
(5d), and to see the subtle ambiguity in (1-2) as reflecting the same kind of difference between judgment forms.

It raises the question of whether there is a derivation of Milsark's generalization directly from the assumptions involved in the ontology underyling the thetic categorical distinction. It is the goal of this paper to explore such a derivation.

Consider how it would go:

11. Derivation of Milsark's Generalization (First version)

a. ILPs must be predicates in Categorical Judgments.

b. The subject of a categorical judgment cannot be a nonspecific indefinite, its reference is "presupposed".

c. Strong construals are the result of this "presupposition".

d. Therefore, the subject of an ILP must have a strong construal.

(11d) is Milsark's generalization. So to the extent to which (11a-c) can be justified in terms of this type of ontology, there is a derivation of the interaction between strong construals and individual-level predicates.

\section{More Brentano}

Brentano saw his task as giving a classification of cognitive acts. He categorized such 'thoughts' into three main groups: presentations, judgments, and love. By this unexpected latter term, he meant affectual attitudes.

The notion of 'presentation' in his system is both fundamental and difficult to grasp. Its essence is the idea that a cognitive agent can have a presentation of an object without it being the presentation of any particular object. One can entertain presentations of objects which do not nor could not have any real existence, like unicorns, golden mountains, and I suppose round squares. Achieving a presentation of something is the first step toward the other two types of acts, of which judgments concern us most.

Judgments (and affectual attitudes or reactions) come in contrary pairs of affirmation and denial, the two modes of judgment. The basis for a judgment is a simple or compound presentation, so the root of the thetic/categorical distinction is in the definition of possible bases for judgments.

The basis for a thetic judgment is a presentation of an object: an entity or eventuality. An affirmation of such a presentation commits the judger to the existence of something which satisfies the presentation; a denial by contrast expresses a negative existence judgment.

The basis for a categorical judgment is compound: first a presentation which is clarified into a particular object satisf ying the description, and then a property to be affirmed or denied of the object.

Despite the conceptualist foundation of this system, I think we can model it with some familiar objects. Let us equate the notion of a presentation of an object for a description of an object. A description is something which can be satisfied by an 
object. Take object to range over both individuals and eventualities, and we have two sorts of descriptions: descriptions of an individual and descriptions of an eventuality. Properties we can take as basic, but crucially not as descriptions of an individual or an eventuality. The modified ontology then is summarized in (12):

12. Modified Brentanan Ontology

objects: individuals, eventualities

descriptions of individuals and descriptions of eventualities

properties

The assumptions about the forms of judgment can be summarized in (13):

\section{Judgment structure}

a. Basis for a thetic judgment: a description

b. Basis for a categorical judgment: an object and a property.

c. A thetic judgment is an affirmation or denial of the description in the basis. (Existential commitment)

d. A categorical judgment is an affirmation or denial of the basis property to the object in the basis. (Predication)

Note that (13c) and (13d) contain a theory of negation which recognizes both a relational and a unary notion of negative mode of judgment. The utility of this distinction has been argued for eloquently in precisely the same tradition by Horn 1989 , which put me onto this line of thinking.

We are almost ready to revisit the derivation of Milsark's generalization. What is missing suggests another borrowing from Brentano. If (9) and one reading of (1-2) are thetic judgments, they contain a single description in their basis. What is that description? It is the description of an eventuality of a cat sleeping there or unicoms entering a garden or a group of people being at a party. But that description is itself a composition of a parameterized eventuality description with various individual descriptions.

Hence we must assume that the theory of argument saturation can work on two levels, either by restricting a parameter in an eventuality description with another description or specifying an object as the value of the parameter.

In Brentano's view of the existential commitment of a thetic judgment, only one description is affirmed. In (9), only the existence of the eventuality is affirmed, but commitment to that description will indirectly, obliquely in his translator's terms, commit the judger to the existence of the cat. But the cat has a much fuzzier existence in the thetic judgment than it does in the categorical version, where it is presented and affirmed directly, first of all, before moving on to the predication. In this way, Brentano's view of the thetic mode of judgment nicely represents the effects of unselective existential closure over the description, giving priority to one object, while obliquely entailing the existence of subparts of the complex object.

These assumptions also call our attention to the weak and strong distinction again, suggesting that the essence of a weak construal is interpretation as a description. Descriptions cannot combine with nondescriptions. So if weak construals are descriptions, then the impossibility of combining them directly with properties follows.

But this view fractures Milsark's, and Barwise and Cooper's, cut of weak vs strong. Though the definite descriptions are in their terms strong, they must, 
however, on the view given here count as descriptions of objects which can specify the parameters of complex descriptions. So these strongly construed phrases are not banned from inclusion in the basis of a thetic judgment. The quantificational ones are another matter, and we will turn to them shortly.

But first let us return to the attempt to derive Milsark's generalization from this analysis:

11. Derivation of Milsark's Generalization (Second Try)

a. Properties cannot be the basis of a thetic judgment, only part of the basis of a Categorical Judgment.

b. The subject of the basis of a categorical judgment must be an object, not a description of an object.

c. Strong construals are either object denoting or quantificational.

d. Therefore, the subject of an ILP must have a strong construal.

We have cleaned up the derivation a bit, but highlighted the question of how general propositions are to be analyzed. Before tuming to this, there are some loose ends and connections to be acknowledged.

\section{Some loose ends and connections}

One loose end is, how is it possible to have a categorical judgment whose basis contains a description? That is, how is (10), with the material which can combine to form the basis of a thetic judgment to be factored into an individual and a property? I assume that it is possible to freely derive properties from a thetic judgment by abstraction. That the judgment form is independent of the syntactic notion of subject is made clear by Kuroda's discussion of the result of wa marking on a non subject, as in (15):

15. neko wa inu ga oikakete iru

the cat I the/a dog is chasing

The cat is being chased by a dog.

He says, taking certain liberties with examples and example numbers, (1972:167)

"The judgment represented by (10) is based on the thetic judgment represented by (9); the syntactic subject inu is made the subject of that categorical judgment. Now the syntactic subject is not the only constituent that can be made the subject of a categorical judgment. [... in (15)] one may have a categorical judgment based on [a] thetic judgment but setting [the object] neko as the subject.

I take the idea that properties can be abstracted from thetic judgments to be the mechanism which allows descriptions to provide the predicate of a categorical judgment. The derived property is the property of being a participant in an eventuality of that description.

The analysis laid out here does not contain much which is startlingly novel. Most, if not all, of the distinctions drawn here are part of standard situation semantics: the separation of polarity values out in the specification of the description of a state of affairs, the restriction approach to argument 'saturation', and (catephorically) the importance of the spatiotemporal parameter in certain states of affairs. Drawing out those connections here would take me beyond the time and preparation for this talk. ${ }^{\dagger}$ 
Returning to the Kratzer-Diesing derivation of Milsark's generalization, we can see what I have laid out here as a proposal for interpreting the abstract INFL structures postulated by Diesing's analysis. Taking the points in turn, the weak readings of indefinites are the result of oblique existential closure due to the thetic mode of judgment. If in the languages that she is describing the category VP is crucially the domain of existential closure, it means that thetic judgments draw their basis from material analyzed as originating in the VP. Individual level predicates are not descriptions (and here the role of the Kratzer definition in terms of the presence of an eventuality argument is clear), so they cannot be the basis of a thetic judgment. There is a direct analogy between the unary nature of a thetic basis and hence mode of judgment and the binary nature of the basis and mode of a categorical judgment. The possibility of derived properties means that stagelevel predicates can occur in categorical judgments (i.e. 'under transitive INFL') as well. Reconstruction is simply an artifact of the assumptions about the syntaxsemantics interface. But the derivation of the conclusion seems quite parallel. Hence the literature on the categorical and thetic judgments, and particularly Kuroda's work, is relevant reading for those interested in expanding her account or in the line of analysis of Bowers 1993.

I have not gone into the derivation of the correlated effects in existentials, because I have nothing to say beyond what McNally 1992 says. She bases her analysis on the notion that the existential construction involves the affirmation or denial of a description in the pivot position and makes a case for dividing the Barwisian-Cooperian category of strong DPs in the way that has been forced on us here.

\section{Quantificational Readings Revisited}

So in the remaining time let I will turn to matters of extension and address first the analysis of quantificationally strong DPs.

Brentano suggests that the thetic/categorical distinction ought to run through the domain of quantificational propositions as well. As Kuroda points out, the details of that analysis are motivated by other than descriptive considerations and his proposals should not be imported into the current situation. As far as I know, the binding operator analysis of generalized quantifiers or the higher-order relational interpretation of quantificational determiners could be imported into this view. What I want to do here is sketch an analysis, without arguing for it now, of another possibility created by the notion of 'mode of judgment' in this analysis.

As described here, the algebra of the two modes of judgment is the simplest boolean structure: two dual values of negation and affirmation. But we might consider expanding this algebra further to include the modes corresponding to quantificational determiners. If we did this, an obvious analogy between the tripartite structure of quantificational operator representations and the tripartite (including the mode of judgment) nature of a categorical judgment springs to mind. The analogy would match the restriction of a quantificational operator with the subject of the categorical judgment and the nuclear scope, properly abstracted over the variable to be bound with the predicate of the judgment.

Now what do we know about the subject of a categorical judgment? It is presupposed, in the sense that 'the mind is directed first to the object which is the subject, and then the predicate is affirmed or denied of the subject.' Now restrictions of quantificational operators are known for their presuppositional nature. They are presupposed to be non-empty in the usual case; the domain of quantification is defined first and then the elements of the domain are subjected 
individually to the nuclear scope and checked for satisfaction. This has a pleasing parallel with the description of a categorical judgment and reminds us again that it is the direct successor to Aristotle's categorical analysis of quantification.

Certain determiners, particularly the proportional ones, are essentially relational. But not all are. The weak determiners are equivalent to predicates on sets. There is no necessity in treating them as essentially relational, though no barrier to that analysis either.

But if we imagine generalizing the notion 'mode of judgment' here, we are led to ask whether there would be thetic quantificational sentences as well. How would they differ from categorical quantificational sentences? They would show none of the presuppositional effects in their restrictions that are due to the presuppositional nature of the subject of a categorical judgment. Can we test this conjecture out?

I think we can, though I can offer only two suggestions, one autobiographical. I got into this state from concerns that arose in my analysis of negative concord within the theory of indefinites. I proposed at this conference two years ago an analysis in which negative concord terms were treated as indefinites roof ed in the nuclear scope of an abstract negation operator associated with the head of the clause, a feature of INFL. In LF-movement based analyses of negative concord, such as Laka (1990) and Zanuttini (1991), a similar approach is taken but the assumption is that the negative concord terms move into the specifier of a negative operator. Identifying the specifier of a negative head with the restriction of a negative operator, I realized that these potentially represented two different approaches to negative concord, one which claimed the negative concord was essentially quantificational and the terms represented the restriction of the operator (the LF movement account) and a non-quantificational account (mine) in which the terms were just weakly construed descriptions in the scope of the abstract negative operator.

Of course the laws of quantifier negation render the two approaches truthconditionally equivalent. So I had to go in search of a probe which would distinguish the two positions. And I found the presuppositional nature of the restriction of a quantificational operator. What is at issue is the semantic analysis of a sentence like (16), even in standard English.

\section{Nobody left}

Should this be taken as a quantificational claim, presuming that there are people and claiming of that set of people that none of them left? Having trained myself to spot the subtle difference between categorical and thetic judgments, however, I convinced myself that while it could be taken that way, it needn't. I can imagine perceiving a situation in which nothing much happens. In particular, no exitings occur. And then I could use (16) to describe that situation. It sounds like the way you describe the use of a sentence expressing a thetic judgment to me. But consider the sentences in (17)-(19).

\section{Most people left \\ 18. Each person left \\ 19. Three people left}

The determiner most is essentially relational, and so we know that (17) must express a categorical judgment. And indeed it seems that you cannot use (17) without presupposing a particular set of people about which you are making the claim that most left. (18) contains the distributive universal each and the same 
intuition holds. Now (19) contains a weak determiner, so it is actually ambiguous between a categorical and a thetic mode of predication (as well as having a description construal). And I think that it is possible to see it as ambiguous as well, between a presuppositional reading which is covertly partitive (the categorical construal) and the nonpresuppositional reading on which no particular set of people is presumed. Of course the description analysis of the weak determiner phrases has already provided an account of this reading as well, so there is no argument here for a thetic mode of judgment analysis of it. But I would have to work to prevent it.

Finally let me note that as far as I can tell, the creation of a generalized conversational scalar implicature from these determiners correlates exactly with the presence of a categorical construal. The proportional quantifiers always give rise to it, as they are always covertly partitive. But on the thetic reading of (19) (with no sentence accent in the subject phrase) there is no implicature that there were people who didn't leave. However on the categorical construal, with a sentence accent in the subject phrase, there is one. This is an effect I can't see deriving from the simple truth-conditional notion of a proposition.

\section{Conclusion}

There are more points of extension and connection than I have time to lay out here. So I will conclude just by reviewing the original goal of the paper: to investigate the extent to which the Milsarkian effects were derivable from the Kurodian assumption that the thetic / categorical distinction should be imported directly into the semantics, rather than considered only an aspect of discourse information packaging, where a whole literature on the distinction exists that has not been reviewed here. (But cf. Sasse 1987. The principal argument for seeing this distinction as part of the semantic foundation is that (perhaps universally) certain attempts to create a proposition simply fail, and they fail in embedded contexts as well as root contexts.)

I judge the attempt to have been successful to the extent that it has allowed us to answer the questions posed above.

20.a Keep the distinction between ILPS and SLPS as one between properties and descriptions, making use of Kratzer's proposal if possible.

.b Weak and strong construals of DPs are not the distinction that Milsark originally made nor that of Barwise and Cooper, but correspond to a description, referential expression, and quantificational mode of judgment contrast which is epiphenomenal on the underlying distinction between judgment types.

.c Milsark's generalization is derived as above.

.d It is possible for a language to have lexical items which are able to be interpreted as descriptions or referring expressions, but not modes of predication.

.e They both are. 
Notes

*This is the SALT talk as presented. I am grateful to Donka Farkas, Ted Fernald, Yuki Kuroda, Louise McNally, Peter Svenonius, and particularly Barbara Scholz for discussions of these ideas; none of them are responsible for the result. This research was supported by NFS grant BNS -9021398.

$\dagger$ Some of these issues have been clarified for me in post-SALT conversations with Robin Cooper; the results of those discussions have not been incorporated here.

\section{References}

Barwise, Jon and Robin Cooper. 1980. Generalized quantifiers and natural language. Linguistics and Philosophy 4:159-219.

Bowers, John. 1993. The syntax of predication. Linguistic Inquiry 24:591-656.

Brentano, Franz. 1973. Psychology from an empirical point of view. Translated by Antos C. Rancurello, D. B. Terrell, and Linda L. McAlister from Psychologie vom empirischen Standpunkt $(1874,1924)$. Sections V-IX.

Carlson, Gregory N. 1977. Reference to Kinds in English. $\mathrm{PhD}$ dissertation, UMass/Amherst.

Diesing, Molly. 1992. Indefinites. Cambridge, MA: MIT Press.

Horn, Laurence A. 1989. The natural history of negation. Chicago: University of Chicago Press.

Kratzer, Angelika. 1989. Stage-Level and Individual-Level predicates. UMass MS.

Kuroda, S.-Y. 1972. The categorical and the thetic judgment. Foundations of Language 9:153185.

Kuroda, S.-Y. 1992. Japanese syntax and semantics. Dordrecht: Kluwer.

Laka, Itziar. 1990. Negation in syntax: On the nature of functional categories and projections. $\mathrm{PhD}$. dissertation, MIT.

McNally, Louise. 1992. An inter pretation for the English existential construction. $\mathrm{PhD}$ dissertation, UC Santa Cruz.

Milsark, Gary. 1974. Existential sentences in English. PhD dissertation, MIT.

Sasse, Hans-Jürgen. 1987. The thetic/categorical distinction revisited. Linguistics 25:511-580.

Zanuttini, Raffaella. 1991. Syntactic Properties of Sentential Negation: A Comparative Study of Romance Languages. University of Pennsylvania, Ph.D. Dissertation.

Cowell College

University of California

Santa Cruz, CA 95064 USA

ladusaw@ling.ucsc.edu 